\title{
Le test des gestes et de la parole dans la
} communication politique télévisée

Interview de Jean MOUCHON

Denis Muzet

\section{OpenEdition \\ Journals}

Édition électronique

URL : http://journals.openedition.org/edc/2868

DOI : $10.4000 /$ edc. 2868

ISSN : 2101-0366

Éditeur

Université Lille-3

Édition imprimée

Date de publication : 1 mai 1989

Pagination : 125-129

ISSN : 1270-6841

Référence électronique

Denis Muzet, «Le test des gestes et de la parole dans la communication politique télévisée », Études de communication [En ligne], 10 | 1989, mis en ligne le 12 février 2012, consulté le 20 avril 2019. URL : http://journals.openedition.org/edc/2868; DOI : 10.4000/edc.2868

Ce document a été généré automatiquement le 20 avril 2019

(c) Tous droits réservés 


\title{
Le test des gestes et de la parole dans la communication politique télévisée
}

Interview de Jean MOUCHON

\author{
Denis Muzet
}

1 En communication contemporaine on doit tenir compte des réactions d'un public large et hétérogène.

Quelles perspectives et quelles méthodes les Instituts d'études retiennent-ils pour être en phase avec des messages composites où la parole côtoie l'image et le geste?

La réponse de Denis Muzet, directeur de l'Institut Médiascopie...

2 J.M. Pourquoi avoir créé l'Institut Médiascopie alors qu'il existe déjà de nombreux Instituts d'études d'opinion?

3 D.M. Les Instituts de sondage, et d'une façon générale les Instituts d'opinion, n'ont pas jusqu'à présent pris en compte la dimension émotionnelle dans l'impact des messages, qu'il s'agisse de la communication publicitaire ou du discours politique. Jusqu'à présent, l'approche mise en oeuvre est d'ordre quantitatif, elle vise à mesurer la mémorisation et à comptabiliser les opinions déclarées. Pour cerner l'impact d'une communication on soumet un film ou une émission au public et, ensuite, à travers le recueil de déclarations verbales on reconstitue les perceptions, on dégage ce qui a été retenu. Cette approche traditionnelle a beaucoup d'inconvénients. D'abord elle passe par le verbe, par le recueil des opinions et leur interprétation. Mais on sait très bien qu'entre le ressenti effectif d'un message à un moment donné et l'opinion déclarée il y a des écarts importants. Tous les téléspectateurs ne sont pas à même d'exprimer leur opinion avec clarté ou d'être fidèles à ce qu'ils ont vécu et, par ailleurs, le langage est toujours relativement ambigu. Il existe donc un certain nombre de problèmes de décodage et d'interprétation dès lors qu'on travaille sur du verbe.

Une deuxième difficulté tient au fait que quand on interroge des gens après une émission la mémoire intervient. Or, autant il peut être important de savoir le travail de sélection qu'elle a réalisé, autant il paraît essentiel de voir, avant ce filtrage, ce que les gens ont ressenti dans "l'épaisseur du présent », dans l'instantané de la communication. L'échelle pertinente étant à mon 
avis plutôt de l'ordre de la seconde ou des 5-10 secondes que de l'ordre de la globalité.

Enfin, troisième phénomène lié au précédent, l'interrogation après coup révèle une perception globale et l'on ne sait pas précisément en quoi chaque composante du discours a pu déterminer l'impact de la globalité du discours. La limite fondamentale est que le témoignage est recueilli après exposition complète.

J.M. Quelle réponse la médiascopie apporte-t-elle à ces défauts de méthode?

5 D.M. Par rapport à ces limites méthodologiques nous nous sommes dit qu'il fallait essayer de restituer la dimension instantanée, linéaire, continue, la dimension en temps réel et surtout la dimension émotionnelle. Quand l'on s'attache à évaluer l'impact instantané d'une communication, l'on est obligé de s'attacher à prendre en compte l'aspect subjectif de la relation intime qui se crée entre l'émetteur et le récepteur. Cette relation est uniquement subjective parce que les messages se complexifient et que l'image, la parole, le geste jouent un rôle de plus en plus important. On n'est plus en présence d'un message purement dénoté, purement didactique où il n'y aurait qu'un problème de compréhension rationnelle, il s'agit de messages composites dans lesquels les codes se superposent et où l'on trouve une grande densité d'informations comme dans les vidéoclips ou dans la publicité. Compte tenu de cette complexité nouvelle il faut donc essayer d'avoir une approche qui privilégie non pas l'opinion mais ce que j'appelle la réaction, c'est-à-dire la dimension spontanée et qui fasse la part de ce qui est de l'ordre du cognitif et de ce qui est de l'ordre plutôt de l'émotif. J.M. Quel dispositif vous permet-il de suivre la réaction des récepteurs?

7 D.M. Le dispositif est simple: c'est un petit appareil - nommé médiascope - qui comporte un curseur linéaire évoluant de haut en bas et qui permet à tout moment au répondant d'émettre un jugement, une réaction instantanée sur un critère qui peut varier mais qui est en général «j'apprécie/je n'apprécie pas» sur des messages publicitaires ou des émissions de télévision, et, "d'accord/pas d'accord» sur des discours politiques, notamment. Dans ce dernier cas la consigne consiste à dire:» plus vous êtes d'accord avec ce que vous voyez et entendez plus vous poussez le curseur vers le haut et moins vous êtes d'accord plus vous le poussez vers le bas "; sachant que le mouvement de curseur va générer selon la position une note de 0 à 10 qui est saisie par des moyens informatiques, traitée instantanément puis visualisée sous forme de courbe correspondant à la durée du message. La saisie de réactions médiascopiques auprès d'un échantillon est précédée d'un entraînement selon un protocole extrêmement rigoureux qui permet aux gens d'apprendre le maniement du curseur en continu et en temps réel.

J.M. Comment est constituéle public-échantillon?

9 D.M. Par exemple pour une émission politique, "L "Heure de vérité" ou "Questions à domicile ", l'échantillon est généralement de 50 personnes, réparties selon des quotas précis, moitié hommes/ moitié femmes, moitié 18-35 ans/moitié 36-60 ans, catégories sociales diversifiées, opinions politiques contrastées avec 1/3 favorables à la majorité, 1/3 d'indécis et 1/3 favorables à l'opposition.

10 J.M. Pour autant la médiascopie est-elle exclusive des autres approches. ?

11 D.M. Non elle n'est pas exclusive et ne se substitue pas aux autres approches. Elle est complémentaire: une fois qu'on aura réalisé la médiascopie pour saisir les phénomènes que j'évoquais antérieurement, on fera suivre l'émission ou le programme de toute une série d'interviews, soit individuels soit de groupes, afin de prendre en compte la dimension de la mémorisation, de la compréhension et de la perception globale. En fait on va travailler en croisant les données de la médiascopie avec les données globales. Pour être complet, l'interprétation des réactions qu'on observe en médiascopie se fait de trois manières. La première voie d'interprétation est la voie interne, celle de la médiascopie. On analyse les courbes, on observe tel ou tel phénomène 
d'adhésion ou de rejet à tel ou tel moment et on s'interroge sur ce qui a pu causer cette réaction. Comme le message vidéo originel est mixé aux courbes et que le délai de réaction est de l'ordre de la seconde, il ne sera pas très difficile de constater, de façon empirique, que c'est telle phrase, telle attitude ou tel mot important qui a suscité telle réaction. Donc au premier degré on analyse la courbe en fonction du contenu grossier du message, c'est le niveau intrinsèque. La deuxième voie est celle de l'opinion. En interrogeant les gens après coup nous mettons en rapport leur réaction et leur opinion. Il y aura à la fois convergence et décalage, convergence car il est bien rare qu'il y ait une contradiction totale entre le ressenti instantané des gens et leur verbalisation - et, parfois, décalage -. Les décalages sont très intéressants. Par exemple, lors d'une enquête réalisée pour le Nouvel Observateur sur les enfants et la télévision, nous avions fait la médiascopie des instituteurs de ces enfants-là. Leur réaction et leur opinion marquèrent un écart important à propos de la playmate - cette femme qui se déshabille le samedi pendant quelques secondes dans l'émission de variétés Cocoricocoboy. Leur réaction spontanée traduite par la médiascopie était très positive tandis que le discours consécutif était extrêmement critique! Y a-t-il contradiction? On peut s'interroger, mais il y a en tout cas décalage. La troisième voie d'interprétation est la voie sémiologique. J'aurais pu en parler en premier parce qu'avant de recueillir des opinions et souvent avant même de recueillir des réactions à travers la médiascopie on analyse et on décode le message pour cerner les constituants, quels signes il mobilise, comment ces signes sont organisés. A ce stadelà on fait des hypothèses sur le sens et sur le fonctionnement du message qui aboutit à une typologie. Dans le discours politique ce sera, par exemple, l'analyse du discours et éventuellement l'analyse des gestes et des attitudes. Dans le message publicitaire ce sera l'analyse des signes et des codes. C'est très important car la typologie qui en résulte permet d'identifier assez bien les registres employés et est très souvent prédictive des réactions. A travers notre banque de données, plus on fait le parcours complet c'est-à-dire plus on sait quels sont les langages en vigueur dans un univers, quelles sont les réactions observées jusqu'à présent par rapport à ces langages, quelles sont les perceptions globales qui en résultent exprimées verbalement plus on est en mesure face à un message brut de faire des hypothèses sur sa réception et d'éviter ainsi un diagnostic complet. C'est le cas évidemment dans le domaine politique où les discours sont redondants et où les évolutions de fond de l'opinion sont assez lentes.

12 J.M. Votre banque de données vous permet-elle, par rapport à la communication politique à la télévision, de dégager des règles pour la prise de parole?

13 D .M. Oui, en effet. Je crois qu'il y a quelques règles extrêmement simples. La première règle c'est ce que j'appelle l'entrée directe dans la réponse. Par rapport à une question donnée d'un journaliste, l'homme politique doit répondre - quand il souhaite y répondre car il existe des cas où il n'a pas intérêt à répondre et où il faut utiliser des subterfuges - de la manière la plus directe possible écartant tous les préambules, les préalables, toutes les formules alambiquées typiques de la langue de bois et employées par une certaine génération d'hommes politiques. C'est un problème de génération et de formation rhétorique quasi scolaire. C'est aussi chez certains une manière de ménager un temps de réflexion. Or le préambule a toujours un effet désastreux. Il laisse le temps aux auditeurs de décrocher et il est souvent perçu comme une tentative de contournement de la question, une tentative de refus de répondre. La réponse différée est fréquemment ressentie comme une fuite. Deuxième règle : l'emploi d'un vocabulaire simple, familier, compréhensible. Dans ce sens il faut fuir tous les technocratismes. Parler de demandeurs d'emploi au lieu de chômeurs est inutile. Il faut fuir aussi les raisonnements macroéconomiques, les abstractions, c'est-à-dire parler de telle entreprise en difficulté que l'on connait plutôt que de parler des entreprises en général. Un propos formulé concrètement dans un raisonnement suscite une adhésion très nettement supérieure à l'adhésion qu'on observerait sur le même raisonnement énoncé de façon abstraite et générale. 

parole est bien objet d'apprentissage. En sachant aussi qu'une modification de formulation peut affecter le fond, je dirais que c'est là le danger! Le problème de forme est à relier à l'environnement, aux idées et aux valeurs du moment. Néanmoins, sur la forme seule, il est évident qu'il y a des hommes politiques qui ont moins besoin de travailler, moins besoin de faire appel à une pédagogie. Mais il faut reconnaître quand même que beaucoup ne sont pas encore au point.

J.M. La plupart des leaders politiques font appel à l'Institut Médiascopie, qu'est-ce qu'ils en retirent?

D.M. Nous travaillons en effet pour la quasi totalité des grands leaders, de l'opposition et de la majorité, à quelques exceptions près. Alors est-ce que les leaders - et les challengers d'ailleurs - ont complètement intégré notre démarche, complètement fait leur nos conclusions? Je serai modeste car il y a beaucoup de phénomènes qui entrent en jeu : il y a énormément de conseillers en tout genre autour de chaque personnalité, plus elle monte dans le pouvoir plus elle fait éclore autour d'elle une multitude de conseillers et les moyens des uns ne sont pas ceux des autres, les intérêts des uns ne sont pas forcément ceux des autres. Il y a des phénomènes d'intérêts et des grilles de lecture différentes. L'impact de nos propres investigations est à resituer dans ce contexte et à son niveau mais enfin il n'est pas négligeable...

\section{RÉSUMÉS}

Interview de Denis Muzet, directeur de l'institut Médiascopie, par Jean Mouchon sur la prise en compte de l'image et du geste dans les enquêtes d'opinion menées par les Instituts d'études.

\section{INDEX}

Mots-clés : communication politique, télévision, enquête d'opinion

Keywords : political communication, television, opinion survey

\section{AUTEUR}

\section{DENIS MUZET}

Denis Muzet. Directeur de l'Institut Médiascopie 